\title{
La danseuse prostituée dite " Ouled Naïl », entre mythe et réalité (1830-1962). Des rapports sociaux et des pratiques concrètes.
}

\section{Barkahoum FERHATI}

\section{(2) OpenEdition}

Journals

\section{Édition électronique}

URL : https://journals.openedition.org/clio/584

DOI : 10.4000/clio.584

ISSN : 1777-5299

Éditeur

Belin

Édition imprimée

Date de publication : 1 avril 2003

Pagination : 101-113

ISBN : 2-85816-663-3

ISSN : $1252-7017$

\section{Référence électronique}

Barkahoum FERHATI, «La danseuse prostituée dite « Ouled Nail », entre mythe et réalité (1830-1962). Des rapports sociaux et des pratiques concrètes. », Clio. Histoire, femmes et sociétés [En ligne], 17| 2003, mis en ligne le 27 novembre 2006, consulté le 22 avril 2022. URL : http:// journals.openedition.org/clio/584; DOI : https://doi.org/10.4000/clio.584

Ce document a été généré automatiquement le 22 avril 2022.

Tous droits réservés 


\title{
La danseuse prostituée dite « Ouled Naiil », entre mythe et réalité (1830-1962). Des rapports sociaux et des pratiques concrètes.
}

\author{
Barkahoum FERHATI
}

1 Les conclusions de ma recherche sont fondées sur l'enquête que j'ai menée pendant plusieurs années - dans les archives ${ }^{1}$ et sur le terrain - sur l'évolution du groupe social qui, en Algérie a servi de support à la représentation orientaliste, littéraire et iconographique, de la sexualité vénale. Ce groupe est repérable au moyen d'une reconstruction, au centre de laquelle il y a la «danseuse-prostituée » dite Ouled Naïl ${ }^{2}$, un statut au demeurant problématique, à la fois réel et fantasmé. La cité de Bou-Saâda ${ }^{3}$, terrain de ma recherche, considérée comme une région "impropre ${ }^{4}$ à la mise en valeur coloniale, fut longtemps tenue à distance - relativement bien sûr - de la «civilisation» et de la modernisation. Aussi a-t-elle maintenu tant bien que mal ses caractéristiques propres, supposées archaïques, jusqu'à ce qu'on décide pour elle d'un destin touristique. Le monde des courtisanes et des concubines, celui des «femmes libres » auquel est attaché ce groupe, qu'on a cru faire disparaître avec l'arrêté du 12 août 1830 qui classa les filles publiques en «officielles » et en «clandestines », va resurgir pour se réorganiser autour de la nouvelle économie touristique. Dans ce contexte, comment la société locale s'accommode-t-elle historiquement de ses “déviants", mais aussi s'approprie-t-elle, en même temps qu'elle les rejette, les modes de vies imposés par le système colonial?

Construction d'un espace social de la prostitution

2 La population de Bou-Saâda était traditionnellement formée de plusieurs composantes dont chacune vivait dans son espace propre. Il y avait les Arabes, les Juifs, les Mozabites, les Européens, les Militaires, et enfin les filles publiques, desquelles va émerger le groupe des danseuses dites "Ouled Naïl», celles qui animaient les visites officielles et les fêtes. On envisagera ici son intégration dans le marché du tourisme, en 
prenant en compte plus particulièrement son espace, ainsi que les danses et le costume qui lui étaient propres.

Du café maure à beit el kbira (la grande maison)

3 En 1845, au moment où elle a commencé à être colonisée5, la cité de Bou-Saâda abritait une population de ${ }^{5} 000$ personnes, dont l'activité principale s'articulait autour du commerce des laines. Elle reçut alors une garnison ${ }^{6}$ de plus de 500 hommes. Le soir, les commerçants et les militaires se réunissaient dans les cafés maures pour se distraire. Faidherbe, l'ingénieur du génie militaire qui fortifiait la ville, observait: "Au café maure, des jeunes filles des Ouled Naïl, couvertes de vêtements et d'ornements bizarres, dansent au son de cette étrange musique $»^{7}$.

4 C'était au mezouard, un agent désigné au temps du beyelik (période ottomane), qu'il revenait d'assurer la surveillance des filles publiques et de lever l'impôt. Une institution qui fut reconduite pendant quelques temps par l'administration coloniale. En 1850, jugée archaïque, elle fut remplacée par la police des mœurs ${ }^{8}$. On affecta alors aux filles publiques un lieu spécifique, appelé par Galland «l'Asile des Naïlia » qu'on installa sur la place, bordée d'un côté par les boutiques indigènes, de l'autre par le Commissariat de police et la "maison d'école "9. Tandis que leur visite sanitaire s'effectuait à l'ambulance militaire jusqu'à la réalisation de l'hôpital militaire en 1853. On leur aménagea une salle pour la visite et une seconde pour l'hospitalisation. Le quartier réservé était né. Organisé autour d'une cour centrale entourée de seize à dixhuit cabanons, dont chacun était destiné à loger deux femmes prostituées, il était nommé localement Beit el kbira ${ }^{10}$ (la grande maison); terme qui désigne en arabe une tribu de "grande tente» ou une grande famille. A ce terme est associée une circonspection que la mémoire locale n'évoque qu'avec répugnance. Le terme tabeg el kelb, la patte levée du chien, rappelle l'image abjecte qu'avait engendré ce lieu. Un lieu qui resta, pendant longtemps, au centre de la vie économique et sociale de la cité. Vers les années 1930, on contesta cette localisation, il fut alors déplacé vers les berges de l'Oued de Bou-Saâda. A ce bâtiment on annexa un dispensaire appelé sbitar el hibç (l'infirmerie prison). On se souvient encore à Bou-Saada de l'intransigeance du docteur Nicolaï ${ }^{11}$. Sa réputation y était connue de tous. Les filles publiques ont été définitivement enfermées dans ce nouvel espace, dont elles ne sortaient plus que sur autorisation. On était au début du XXe siècle, au moment de l'émergence de la bourgeoisie puritaine et du réformisme religieux ${ }^{12}$ qui s'affirmèrent avec l'évolution urbaine de la cité.

Des maisons de tolérance à la rue dite « rue des Ouled Naïl »

$5 \mathrm{Au}$ cours des années 1930, la population de Bou-Saada atteint 50000 âmes; l'urbanisation et l'euphorie touristique aidant, la topographie de la prostitution va, dès lors, se modifier. Autour de beit el kabira, installée sur les berges de l'oued, vont s'établir peu à peu des maisons de tolérance. Elles formèrent la rue de la "tolérance ", appelée communément la « rue des Ouled Naïl ». Officiellement, cette rue était baptisée la rue Bosquet, du nom d'un des héros de l'Armée d'Afrique dont on perdit la trace. La mémoire évoque plutôt, $z^{\prime}$ gag el qanqi, la rue de la Lanterne qui fut l'une des premières à être éclairée. En 1952, cette rue était formée d'une vingtaine de maisons de tolérance abritant chacune en moyenne cinq filles prostituées. Chaque " maison » était désignée du terme haush flana, la "propriété » d'une telle. En Islam, il n'est pas exclu qu'une femme, célibataire, épouse, veuve ou divorcée, possède des biens mobiliers et immobiliers dont elle conserve l'entière disposition. Chaque "maison» était donc 
désignée du nom de sa propriétaire, et souvent par son origine tribale. Par exemple, on garde le souvenir de haush Zaïdania el M'dukaniya, une véritable artiste venue d'un village voisin M'Dukan. D'une rare beauté dit-on, elle fut le modèle préféré du peintre orientaliste Etienne Dinet (1864-1929) ${ }^{13}$.

La rue des Ouled Naïl était, à Bou-Saada, la plus animée de la ville. En apparence bien sûr, puisque la rue de l'amour et de la joie était aussi un lieu de mort et de bagarres. Il ne se passait pas un jour sans que la police ou l'armée n'interviennent. Qui ne se souvient de l'émeute de 1932 ? Une bagarre entre clients qui se disputaient les faveurs d'une prostituée dégénéra en une véritable révolte qui souleva presque toute la ville. L'administration centrale avait même cru à une rébellion populaire suscitée par les nationalistes. Le Gouverneur Général y dépêcha une commission pour enquêter ${ }^{14}$. Le lieu de l'amour vénal de nouveau au centre de la cité, les voies y conduisant furent murées. Une manière de dénoncer l'infamie et de s'en distancer. Comme à Alger où faute de disposer d'un espace propre à la prostitution, l'inscription " Maison honnête " au frontispice des maisons indiquait la frontière à ne pas franchir. Ou encore à Tunis, où les portes des filles publiques étaient marquées d'une teinte rouge, tahmir. Mais la visée économique était telle que cette distanciation était quelque peu escamotée. En effet, le syndicat d'initiative insérait la « rue des Ouled Naïl » dans son programme de visite, d'» attraction touristique » et faisait de la maison de tolérance une «maison de danse ». Une mise en scène dans laquelle la fille publique, consciente plus que jamais de son nouveau rôle, mit à contribution tout son savoir-faire.

Reconstitution des « usages » indigènes liées à l'activité prostitutionnelle

7 L'avènement du tourisme, l'exotisme aidant, va conduire à mettre à profit certaines pratiques locales, tandis que la danseuse prostituée acquiert un savoir-faire au contact des touristes. Elle cherche aussi à répondre aux exigences de son temps, non seulement en façonnant le costume «traditionnel » mais aussi en apportant des innovations dans l'exécution de la danse « hiératique ».

M’bita, nuitée de danse

8 Le syndicat d'initiative avait introduit dans ses programmes des soirées m'bita, des attractions essentielles. La m'bita devait être une nuitée de chants et de danses. Le commerce sexuel n'est pas la seule chose visée ${ }^{15}$. Dans la m'bita du Syndicat d'Initiative de Bou Saada, les filles publiques exécutaient, pour les touristes, des danses locales. La danse saâdaoui, dite aussi naili, est accomplie par deux femmes qui vont et viennent en une démarche glissante et légère, scandée par des balancements de bras et des flexions de mains ; puis, continuant à avancer de front, elles unissent leurs mains sur lesquelles elles balancent, en une modulation douce, leurs bras demeurés libres. La littérature qualifiait cette danse de « hiératique $»^{16}$. La carte postale a fixé l'image de la « danse de la bouteille », qui fit son apparition pour la première fois au quartier réservé, où la danseuse portait une bouteille d'alcool en équilibre sur sa tête. Il est une autre danse étrangère à la tradition locale, la «danse nue ». Émile Dermenghem la qualifiait d'hérésie ${ }^{17}$. Elle était exécutée en seconde partie du programme de la soirée, moyennant un supplément. On remarque que, lorsque les demoiselles dansaient nues, les musiciens se retournaient sur leurs tabourets et jouaient le nez contre le mur ${ }^{18}$.

Le costume et ses accessoires

9 La danseuse ne pouvait se présenter à son public et à ses clients qu'affublée du costume "traditionnel $»^{19}$. Un costume qui ne diffère guère de celui des autres femmes. Toutefois, de part son contact avec l'extérieur, elle contribua à son évolution. Le 
règlement de la prostitution n'imposait pas de tenue spéciale à la prostituée, mais il recommandait la "décence ». Il exigeait de cette dernière le port du voile lorsqu'elle était de sortie. Celui-ci est appelé bu'awina, voile d'un seul œil. Il est porté par toutes les femmes de Bou-Saâda. Il couvre entièrement le corps de la femme et ne laisse percevoir qu'un seul œil. Vêtement, coiffure et bijoux de la danseuse prostituée étaient complètement dissimulés sous ce voile. Avant le milieu du XIXe siècle, il s'appelait malhafa, sorte de toge agrafée sur la poitrine par des khulalat, des fibules. La taille est retenue par une ceinture. Ce vêtement va évoluer dans l'après-guerre pour devenir la ruba, une robe toute droite montée avec fronces ou plis qui descendent jusqu'au sol. Cette robe ressemble étrangement à la chemise française du XVIIIe siècle. Sa coiffure, où se mêlent foulards, tresses et bijoux, était large et lourde au début du siècle. Laissant apparaître de grosses tresses enroulées autour des oreilles, c'est le gennûr, le turban. Cette coiffure restera en vogue jusqu'à la fin de la Seconde Guerre Mondiale. La tradition interdit aux femmes de se dénuder la tête. Cette obligation était respectée par toutes les femmes, mais elles allaient peu à peu la transgresser. C'est la 'aqsa, coiffure en biais, dont le volume est allégé. Dans les années 1950, les cheveux en tresses sont jetés sur le dos et le front est cerné par un foulard noué, c'est la shedda. Une partie de la tête est mise franchement à l'air. La danseuse a adopté ces coiffures ou ces façons de faire nouvelles, tout en perpétuant certains éléments antérieurs ; elle les a embellis, en usant de son génie pour être toujours la plus belle, même la tête couverte ! Son but n'est-il pas de séduire ? Le vêtement, avec les différentes coiffures, était orné de bijoux et parfois soutenu par d'autres. L'abondance et la diversité ${ }^{20}$ des bijoux s'expliquent par le fait que ce sont là des biens dont on peut se servir dans les jours difficiles. Mais l'attrait pour cette diversité allait s'atténuer peu à peu. Vers les années 1950, ce sont une série de colliers sur lesquels étaient fixés les louis et les napoléons qui étaient suspendus sur la poitrine. La grâce n'y était plus, quoi que leur nombre indiquait la fortune de la danseuse. Aujourd'hui, certaines anciennes danseuses comptent parmi les notables les plus fortunées de la cité.

10 L'entretien de l'apparence ne s'arrête pas au costume; l'action d'embellissement s'exerce aussi sur le corps. Le hammâm, puis la visite médicale hebdomadaire, étaient obligatoires pour préserver d'une redoutable maladie, el mardh elkbir, la syphilis. Le rituel du hammâm est accompagné des pratiques du henné, du massage, du ponçage, et enfin de l'épilation intime; vient ensuite l'apprétement du visage. L'essentiel du maquillage repose sur le khôl et le $s w a k^{21}$ qui blanchit les dents et rougit les gencives. Le khôl accentue la mise en valeur du regard. L'effet est d'autant plus magique que la danseuse esquissera une ghamza, une œillade, pour signifier son consentement à l'amant qui la sollicite. Ses mains et ses pieds sont rougis au henné. Son visage est dessiné par l'ushâm, tatouage, et à son passage l'odeur du bkhûr ${ }^{22}$ vous enivre de ses effluves. La coquetterie, la coiffure, le maquillage et les cosmétiques renvoient simultanément au tragique de l'éphémère, mais aussi à un "mode d'être essentiellement érotique ». Tout en puisant dans les usages "modernes», ses gestes participent de diverses traditions à la pérennité incertaine.

La société locale indigène face à ses déviantes

11 Quels étaient les mécanismes d'entrée de la femme dans la prostitution et quels étaient les dispositifs envisagés pour l'en sortir?

L'entrée dans la prostitution : recrutement et apprentissage 
12 A Bou-Saâda, le recrutement se faisait dès le jeune âge, au sein de la famille, du village ou de la tribu. L'initiation à la danse, point d'entrée dans la prostitution, exigeait un véritable apprentissage. Fatima el coptana (Fatima la capitaine) était "propriétaire " d'une vingtaine de danseuses qu'elle avait recrutées dans son village. Et Yamina, à la redoutable réputation, avait mis à son service toutes ses sœurs et ses nièces. L'enquête de Dermenghem ${ }^{23}$ concluait qu'il s'agissait là de "prostitution traditionnelle», en opposition à la "prostitution sacrée ${ }^{24}$ longtemps tenue pour être à l'origine de la prostitution en Algérie. L'entreprise «familiale » ainsi bâtie, son chef n'était autre que la dame de maison selon le règlement. Elle agissait en véritable patron face à l'administration qui lui imposait un règlement et exigeait d'elle des taxes. C'est ainsi que l'administration a été confortée dans son rôle, rassurée quant aux aptitudes de ses recrues. La vie quotidienne était organisée selon un calendrier établi par l'administration: les samedis et dimanches étaient réservés exclusivement aux militaires pour éviter tout incident avec la clientèle civile. Le mardi, jour de marché à Bou-Saâda, était réservé aux clients civils, voyageurs et comerçants de passage. Le reste de la semaine les prostituées recevaient surtout les citadins. Le programme d'une journée commençait le soir où, dans une première partie, on assistait à un « spectacle banal ». Et dans une deuxième partie, qui commençait très tard, venait le spectacle "des danseuses nues " ${ }^{25}$. Aucun répit n'était accordé à la fille publique de Bou-Saâda ; elle était également sollicitée par l'administration pour animer les festivités officielles. On ne manquait pas le 14 Juillet, lorsque les filles publiques, dans leurs baçurs, palanquins, défilaient aux cotés des militaires dans les rues de la cité.

Malgré cette vie trépidante, la danseuse s'occupait de sa famille. Son aide se concrétisait souvent dans un placement, dans l'achat d'une maison, d'un jardin ou encore de bêtes ${ }^{26}$ qui permettait à ses proches de vivre. Elle était aussi une mère de famille. Les méthodes contraceptives traditionnelles n'aboutissaient pas toujours; combien d'enfants naquirent dans ces conditions! En général, la danseuse n'hésitait pas à envoyer les siens à l'école; certains ont réussi des carrières en dehors de la prostitution. Pour les filles, le choix était limité : naissant et vivant au quartier réservé, l'avenir était assuré, le "métier » de maman étant le seul modèle à suivre. Quant au garçon, il devait apprendre un métier en relation avec le milieu. Shaush était le métier le plus prisé, le garçon administrait alors les biens de sa mère, de sa sœur ou de ses tantes. La vie dans la prostitution n'était d'ailleurs pas définitive. Des « sorties » étaient possibles.

Les possibilités de « sortie » du cadre de la prostitution

14 Après ce programme chargé, auquel la fille publique était soumise pendant ses années de jeunesse, sa gloire passée; vieillie par le temps ou par l'alcool, le tabac, et toutes sortes de boissons frelatées qui faisaient partie des nouvelles pratiques adoptées au quartier réservé, elle était abandonnée. Avec plus de chance, certaines finissaient tenancières de maison de tolérance. Il arrivait que d'autres, après une nuit agitée d'un rêve prémonitoire, quittassent brusquement le quartier réservé pour prendre le « sentier de Dieu » et mener une vie d'ascète. Elles se constituaient en khaunya, sœurs en Islam, investies de la baraka, un don de dieu aux vertus thérapeutiques. Certaines accomplissaient le pèlerinage à La Mecque, devenaient hajat. On se souvient de celle qui, pour accomplir ce commandement de l'Islam, travailla nuit et jour au métier à tisser pour vendre son produit afin de payer son voyage aux lieux Saints. La fortune qu'elle avait amassée lors de son séjour au quartier réservé était remise à des œuvres 
charitables. Pour les plus jeunes, c'est peut-être un mari qui les attendait. Parfois, elles avaient la chance d'être choisies par un homme riche ou par un amant de cœur pour des épousailles que le prophète Mohammed autorise et même recommande aux croyants. C'est un acte de bravoure qu'un musulman se doit de faire pour sortir une femme de la déchéance. À noter que les non Musulmans sont restés seulement des clients. Dans les années 1930, le cas de Fatima m'rat er rumi, l'épouse du Chrétien, que j'ai pu relever dans mon enquête, reste insolite. Cette union fut très mal reçue par sa famille mais aussi par les autres prostituées.Quoi qu'il en soit, c'est par l'expression khraj-ha mina el qhwa, "un tel a sorti une telle de la prostitution », que ces "épouses » étaient désignées socialement. La connotation de cette phrase en dit davantage sur le mépris que l'on peut porter à ces «épouses " que sur la bravoure de l'homme. Mes informatrices, à Bou Saâda, sont des "épouses mères ${ }^{27}$ qui, de près ou de loin, ont été obligées de recevoir ces « épouses » concurrentes. Elles n'avaient pas d'autre choix que celui de dire leur amertume par cette expression. Quant aux « épouses » concurrentes, leur réinsertion au sein de la société locale n'était pas chose évidente, ni acquise d'avance : elles y étaient admises - ou ré-admises - à travers des rites de passage.

Mais avant fallait-il que l'homme tombe dans une mhna, l'amour fou ? C'est de sortilège qu'il est question. Il faut l'en sortir. Pour ce faire, on fait appel au taleb pour le désenvoûter. Si cela échoue, la femme en question est sommée, par divers procédés, de quitter l'homme. Il arrive que la mhna triomphe, c'est le rasm, le concubinage, une étape de sortie possible du quartier réservé. Une telle réservait à un tel certaines nuits, ce qui ne l'empêchait pas de recevoir ses clients. Lorsque la mhna est encore plus forte, la femme est installée dans une demeure en dehors du cadre spatial et administratif de la prostitution. Il s'agit de la radiation, un article du règlement de cette dernière. Accompagnée d'une gouvernante ainsi élue, elle se réserve exclusivement à son amant. La durée du rasm est variable : elle peut durer toute une vie comme être écourtée en fonction des convenances de l'homme. Cet état peut aussi conduire au z'waj, c'est-à-dire au mariage, au nikah, l'union sacrée, selon la loi coranique. Mais pour y accéder, encore faut-il passer par la 'adda, période de purification dont le but essentiel est de s'assurer qu'il n'y a pas de grossesse en cours. La femme ne devait pas recevoir son futur époux jusqu'à l'expiration de cette période de 40 jours. On peut ensuite procéder au z'waj qui exige la présence d'un Imam, prêtre, qui fera la lecture de la fatiha, une sura du Coran, et de deux témoins. La vie antérieure est clôturée par ce rituel. Et le déplacement vers le foyer conjugal constitue l'étape finale de l'accès au statut de l'épouse que j'ai nommée " seconde épouse de plaisir ».

Il n'existe pas de constante dans le statut réservé concrètement à ces épouses. À l'une étaient confiées des tâches nobles, telles que le tissage et la réception des hôtes, tandis que l'autre était traitée en domestique et une troisième entretenue à part avec une gouvernante. Du reste, elles vont emprunter des savoirs aux épouses, comme le tissage et la cuisine qui constituent un gage de reconnaissance. Conscientes de la fragilité de leurs atouts, elles cherchaient à se replacer dans la société comme bonnes ménagères. Les autres épouses, quant à elles, ne sont pas indifférentes à la façon d'être de leurs nouvelles concurrentes. Elles vont leur emprunter l'art de l'entretien du corps et de la séduction. Ce sont là des échanges qui montrent combien la société locale s'accommode de ses déviantes. Elle n'encourage pas la prostitution qu'elle stigmatise par toutes sortes de méthodes, verbales ou non, mais elle ne l'exclue pas complètement; elle la tient plutôt en respect. 

avait du mal à les comprendre et à les percevoir. Méprisées, parce que représentatives d'une société "arriérée », elles ont été reprises pour le compte et dans le cadre de ce que Jean Louis Flandrin appellait un tourisme "foklorisé ». Ce tourisme reposait, en effet, sur "l'Ouled Naiil»: un mythe solidement échafaudé qui mit en échec la loi française du 13 avril 1946 (dite loi Marthe Richard) relative à la fermeture des maisons de tolérance ${ }^{28}$. Au sein de la société locale, prostituée ou pas, la femme reste marginalisée, qu'elle soit dans la condition d'épouse mère pour assurer la descendance ou dans un statut de femme de plaisir pour que puissent être assouvis les besoins sexuels masculins. Ballottée entre deux sociétés, la femme "indigène" reste donc une laissée pour compte.

\section{BIBLIOGRAPHIE}

BERTHERAND (Dr), 1859, « De la prostitution à Alger », Mémoires divers, Alger, Typo V. Vailland. DERMENGHEM Emile, 1950, Prostitution chez les Oulad Nail, 8X202, AOM.

- 1960 Le pays d'Abel : Le Sahara des Ouled-Nails, des Larbaa et des Amours, Paris, Gallimard.

DOUTE Edmond, 1914, En tribu, Paris, Geuthner.

DUCHENE E.-A, 1853, De la prostitution dans la ville d'Alger, depuis la conquête, Paris, Garnier Frères, Londres, J.-B. Baillière.

EUDEL Paul, 1902, Orfêvrerie Algérienne et Tunisienne, Alger, Jourdan.

FAIDHERBE, 1850-1852, Correspondances avec sa mère, 12X159, AOM.

FERHATI Barkahoum, 1996, Le Costume de la femme Ouled Nail. Inventaire analytique et évolution, DEA, EHESS.

- 2002, Lecture d'histoire sociale de la prostitution en Algérie : Le cas de la prostitution dite Ouled Nail de Bou-Saâda, 1830-1962, thèse de doctorat dirigée par Lucette Valensi, EHESS.

FONTAINE Pierre, 1936, « Ouled Naïl, chez elles », Algéria, n 41, juillet, pp.6-8.

GALLAND Charles (de), 1899, Une excursion à M'sila et à Bou-Saâda, Alger, Ollendorft.

GAUDRY Mathéa, 1960, La Société féminine au Djebel Amour et au Ksel, Alger, Société algérienne d'Impressions Diverses.

KERROU M et M'HALLA M., 1991, « La prostitution dans la médina de Tunis aux XIXe et XXe siècles ", Annuaire Afrique du Nord, n XXX, pp. 350-450.

PEIN Th. (colonel), 1871, Lettres familières sur l'Algérie. Le petit royaume arabe, Alger, Jourdan, rééd., 1893.

MILLOT S., 1920, « Le costume du vieil Alger », L’Afrique du Nord illustrée, n 1, pp. 60-63. 
RAYNAUD M, COLONIEU L, HADIDA E (dr), 1946, « Le problème de la lutte antivénérienne en Algérie ", Rapport présenté au nom de la commission centrale algérienne des maladies vénériennes le 14 décembre 1946, Archives INSP d'Alger.

TURIN Yvonne, 1971, Affrontements culturels dans l'Algérie coloniale. Ecoles, mosquées et religions, 1830-1880, Alger, ENAL.

\section{NOTES}

1.Service des archives de l'armée de terre (SHAT) de Vincennes ; Centre des archives d'Outre-mer (CAOM) d'Aix-en-Provence ; Centre des archives d'Alger...

2.Le terme Ouled Nail est une contraction du mot aw lad Sidi Nail, qu'on peut traduire par les enfants du saint éponyme Sidi Nail. Il désigne la tribu des awlad Sidi Nail dont les territoires s'étendent sur les Hauts Plateaux du Sud algérien dont les montagnes portent le nom, les monts des Awlad Nail.

3.Située à $250 \mathrm{~km}$ au sud d'Alger, elle est une des premières oasis du pays.

4.Rapports des bureaux arabes, 1849-1900.

5.Bou-Saâda instituée commune mixte, a été rattachée successivement aux Territoires Militaires de Constantine puis d'Alger et enfin aux Territoires Civils du département d'Alger pour permettre le développement du tourisme.

6.Pein 1871.

7.Faidherbe 1851.

8.Bertherand 1859.

9.Galland $1899: 53$.

10.D’autres noms lui étaient attribués : haush el ihudi ou Dawud (la propriété du Juif David), du nom de celui qui en était le premier gérant. Ou encore el ' $m$ hal, le magasin, où se commerce l'amour vénal.

11.Le docteur Jacques Nicolaï, nommé médecin de colonisation, était affecté au dispensaire de 1930 à 1962 .

12.En 1931, Ben Badis fonda les Oulémas qui prônaient un Islam réformiste.

13.Il vécut, plus de quarante ans, à Bou-Saâda qui lui consacra un musée dont l'auteur a été la directrice jusqu'à son incendie criminel en 1995.

14.Rapports de l'administrateur de Bou-Saâda 1932. Les archives mentionnent des manifestations du même genre dans différentes villes d'Algérie. Le quartier de la prostitution constituait un point névralgique qui peut nous renseigner sur l'état moral, politique et économique de la cité.

15.Kerrou et M'halla, 1993 : 207.

16.Fromentin 1853.

17.Dermenghem $1960: 82$.

18.Une image fixée sur la toile « Les danseuses Ouled Naïl » par le peintre algérois Irriéra.

19.Ferhati 1996.

20.Millot $1920: 21$.

21.Ce sont des soins effectués au moyen d'un bâtonnet d'écorce de noyer.

22.Le bkhûr est un mélange d'essences utilisé par fumigation.

23.Dermenghem 1960.

24.Douté 1904 .

25.Dermenghem $1960: 83$. 
26. Mathieu et Maury $1952: 122$.

27.Ce sont celles dont les épousailles supposent des alliances entre groupes. Bnt el'a m, la cousine, est la plus privilégiée.

28.Ferhati, La Réglementation de la prositution en Algérie des enjeux en sousbassement, 1830-1962 titre provisoire de l'ouvrage à paraître en 2003, aux éditions Bouchène.

\section{RÉSUMÉS}

Cet article traite des rapports entre colonisés et coloniaux à travers un sujet tabou: la prostitution. Une pratique qui perturbe l'ordre social de la société coloniale que l'on veut morale selon ses propres référents. Elle se dote d'un certain nombre de moyens pour maîtriser, canaliser et surveiller cette pratique et pour cause les maladies vénériennes qui hantaient les esprits. Jusqu'au moment où la raison économique, celle du tourisme notamment, va l'emporter. «L'Ouled Naïl » (un terme générique qui englobait des statuts de courtisane, concubine, danseuse et prostituée) va répondre à cette demande.

Un ensemble de statuts ou de rôles bien précis dans la société colonisée : le passage de l'un à l'autre s'effectue à travers des rituels que la société coloniale ne pouvait percevoir.

The dancer prostitute known as «Ouled Naïl», between myth and reality (1830-1962). Social relationship and real practices : this article deals with the relationship between colonised people and colonial staff through the taboo issue of prostitution, a practice that disturbed the social order of the colonial society, which, according to its own referents, was supposed to be moral. Through a wide range of means, colonial society controlled, channelled and supervised prostitution, supposedly because of the venereal diseases which haunted the minds. This lasted until the economic reason prevailed, in particular tourism. The "Ouled Nail » (a generic term which included the status of courtesan, concubine, dancer and prostitute) fulfilled this need. Each status or role being quite specific in the colonised society, where the passage from one to another was carried out through rituals that the colonial society could not perceive.

\section{AUTEUR}

\section{BARKAHOUM FERHATI}

Barkahoum FERHATI, architecte diplômée de l'EPAU d'Alger, docteure en Histoire et Civilisation de l'EHESS (Paris), a dirigé le Musée National Etienne Dinet de Bou-Saâda (Algérie) jusqu'à son incendie en août 1995. Aujourd'hui, chercheure au Centre National de Recherches Préhistoriques, Anthropologiques et Historiques, CNRPAH, à Alger, et Commissaire de l'exposition du peintre orientaliste «Etienne Dinet » au musée des Beaux Arts de Nantes (du 17 Octobre 2003- 15 Janvier 2004), Algérie 2003 en France. Elle va publier : La Réglementation de la Prostitution en Algérie pendant la période coloniale. Des enjeux en soubassement, (titre provisoire) 1830-1962, Bouchène, Paris, 2003 et Le musée Etienne Dinet. Genèse d'une création, 1930-1993, Barzakh, Alger, 2003. 